\title{
Molecular characterization of an anthocyanin-related glutathione $S$ - transferase gene in Japanese gentian with the CRISPR/Cas9 system
}

Keisuke Tasaki ${ }^{1,2}$, Momo Yoshida $^{3}$, Minori Nakajima $^{3}$, Atsumi Higuchi ${ }^{1}$, Aiko Watanabe $^{1}$ and Masahiro Nishihara ${ }^{*^{*}}$ (I)

\begin{abstract}
Background: The blue pigmentation of Japanese gentian flowers is due to a polyacylated anthocyanin, gentiodelphin, and all associated biosynthesis genes and several regulatory genes have been cloned and characterized. However, the final step involving the accumulation of anthocyanins in petal vacuoles remains unclear. We cloned and analyzed the glutathione S-transferases (GSTs) in Japanese gentian that are known to be involved in anthocyanin transport in other plant species.

Results: We cloned GST1, which is expressed in gentian flower petals. Additionally, this gene belongs to the Phitype GST clade related to anthocyanin biosynthesis. We used the CRISPR/Cas9-mediated genome editing system to generate loss-of-function GST1 alleles. The edited alleles were confirmed by Sanger and next-generation sequencing analyses. The GST1 genome-edited lines exhibited two types of mutant flower phenotypes, severe (almost white) and mild (pale blue). The phenotypes were associated with decreased anthocyanin accumulation in flower petals. In the GST1 genome-edited lines, sugar-induced stress conditions inhibited the accumulation of anthocyanins in stems and leaves, suggestvhing that GST1 is necessary for stress-related anthocyanin accumulation in organs other than flowers. These observations clearly demonstrate that GST1 is the gene responsible for anthocyanin transport in Japanese gentian, and is necessary for the accumulation of gentiodelphin in flowers.
\end{abstract}

Conclusions: In this study, an anthocyanin-related GST gene in Japanese gentian was functionally characterized. Unlike other biosynthesis genes, the functions of GST genes are difficult to examine in in vitro studies. Thus, the genome-editing strategy described herein may be useful for in vivo investigations of the roles of transport-related genes in gentian plants.

Keywords: Anthocyanin, CRISPR/Cas9, Flower pigmentation, Genome editing, Gentian, GST

\footnotetext{
* Correspondence: mnishiha@ibrc.or.jp

'Iwate Biotechnology Research Center, 22-174-4 Narita, Kitakami, Iwate 024-0003, Japan

Full list of author information is available at the end of the article
}

(c) The Author(s). 2020 Open Access This article is licensed under a Creative Commons Attribution 4.0 International License, which permits use, sharing, adaptation, distribution and reproduction in any medium or format, as long as you give appropriate credit to the original author(s) and the source, provide a link to the Creative Commons licence, and indicate if changes were made. The images or other third party material in this article are included in the article's Creative Commons licence, unless indicated otherwise in a credit line to the material. If material is not included in the article's Creative Commons licence and your intended use is not permitted by statutory regulation or exceeds the permitted use, you will need to obtain permission directly from the copyright holder. To view a copy of this licence, visit http://creativecommons.org/licenses/by/4.0/. The Creative Commons Public Domain Dedication waiver (http://creativecommons.org/publicdomain/zero/1.0/) applies to the data made available in this article, unless otherwise stated in a credit line to the data. 


\section{Background}

The blue flowers of cultivated Japanese gentians are due to the accumulation of a polyacylated anthocyanin, gentiodelphin [delphinidin 3-O- $\beta$-D-glucosyl-5-O-(6-O-caffeoyl- $\beta$-D-glucoside)-3'-O-(6-O-caffeoyl- $\beta$-D-glucoside)], which is a major pigment [1]. Additionally, intramolecular copigmentation is responsible for this blue coloration and pigment stabilization [2,3]. To clarify the molecular mechanism mediating the development of blue gentian flowers, the genes involved in the associated metabolic pathway have been isolated and characterized. These include biosynthesis genes encoding chalcone synthase $(C H S)$, chalcone isomerase $(C H I)$, dihydroflavonol 4reductase $(D F R)$, flavanone 3-hydroxylase $(F 3 H)$, flavonoid 3 '-hydroxylase $\left(F 3^{\prime} H\right)$, flavonoid $3{ }^{\prime}, 5^{\prime}$-hydroxylase $\left(F 3^{\prime} 5^{\prime} H\right)$, flavone synthase II (FNSII), and anthocyanidin synthase $(A N S)$, as well as modification genes, such as those encoding anthocyanin 5-glucosyltransferase (5GT), 3'-glucosyltransferase $\left(3^{\prime} G T\right)$, and 5,3'-aromatic acyltransferase (5/ $\left.3^{\prime} A T\right)$, and regulatory genes encoding MYB and bHLH transcription factors. The transcriptional regulation of flower petal pigmentation has been analyzed in previous investigations involving natural mutant gentian cultivars/lines [4], the overexpression of a chimeric repressor (e.g., SRDXGtMYB3) via stable transformation [5], and a viral vector [6]. The transcriptional regulation of gentians determined with these approaches have been reviewed in detail [7]. The information was also applied to develop PCR-based molecular markers for distinguishing plants that produce white or pink flowers from those that produce blue flowers $[8,9]$. The early selection of progeny based on these molecular markers may be useful for promoting Japanese gentian breeding [10]. However, to the best of our knowledge, the gentian genes related to anthocyanin transport have not been characterized.

In higher plants, anthocyanins biosynthesized in the cytoplasm are transferred to vacuoles via several pathways related to transporters such as the ATP binding cassette $(\mathrm{ABC})$ and glutathione $S$-transferase (GST). Pioneering studies revealed that maize Bronze2 (Bz2) and petunia Anthocyanin9 (An9), which encode GSTs, are responsible for the vacuolar accumulation of anthocyanins [11]. Orthologous GST genes related to anthocyanin accumulation have been identified in several ornamental flowering plants, such as DcGSTF2 in carnation [12] and CkmGST3 in cyclamen [13], but in relatively few other species. Although the genes encoding putative GST and ABC proteins responsible for anthocyanin transport have been detected in many plant species and are available in public databases, only a few have been functionally characterized. One of the reasons for this is that analyzing anthocyanin transport is difficult in vivo. Unless mutant lines are available, confirming gene functions is difficult and the transport of anthocyanin pigments to vacuoles has been examined mainly in in vitro uptake assays with artificial membrane vesicles. Some studies involving the genetic complementation of the Arabidopsis $t t 19$ mutant line revealed potential functions of GSTs [13-16], but it remains unclear whether the GSTs are indeed functional in the original plant species. Virus-induced gene silencing is also useful for analyzing gene functions, but it is often difficult to apply this system to horticultural crops and a family of highly homologous genes.

The clustered regularly interspaced short palindromic repeats (CRISPR)/Cas9 system is the most promising genome editing tool in higher plants $[17,18]$, and novel CRISPR technologies are expected to make important contributions to plant science research [19]. The CRIS $\mathrm{PR} / \mathrm{Cas} 9$ system has been used to investigate several ornamental flower species by targeting specific genes such as DFR in Japanese morning glory (Ipomoea nil) [20] and $F 3 H$ in torenia [21]. Additionally, we were the first to establish a CRISPR/Cas9 system targeting phytoene dehydrogenase $(P D S)$ to examine gentians [10]. Moreover, we completed an in vivo functional analysis of anthocyanin modification genes, including 5GT, 3'GT, and 5/3'AT [22]. Thus, the CRISPR/Cas9 system can now be used to accelerate functional analyses of genes in gentians, which are non-model plants.

The objective of this study was to identify an anthocyanin-related GST gene in Japanese gentians and characterize its function via genome editing with a CRISPR/ Cas9 system. A gentian GST gene (GST1) was cloned and confirmed as responsible for anthocyanin accumulation. We demonstrated that this gene influences the accumulation of anthocyanins in both gentian flowers and leaves. To the best of our knowledge, this is the first report of the application of a CRISPR/Cas9 system to study anthocyanin transportrelated genes in higher plants. Furthermore, our findings confirm that the genome-editing strategy applied in this study is useful for identifying uncharacterized gentian genes.

\section{Results \\ Isolation and identification of a GST gene related to anthocyanin pigmentation}

Full-length sequences of two alleles of a deduced GST gene were determined by sequencing the PCR products amplified from cDNA samples derived from gentian cultivar 'Albireo' petals. The coding region sequences of the deduced GST were $642 \mathrm{bp}$ long, with three single nucleotide polymorphisms (SNPs) between the two alleles, which both encode proteins consisting of 214 amino acid residues, with one amino acid difference at position 114 (GST1-1, phenylalanine; GST1-2, leucine). We named these alleles GST1-1 (accession no. LC536038) and GST1-2 (accession no. LC536039), and hereafter refer to them together as GST1. The raw sequencing data are available in the DDBJ Sequence Read Archive 
(DRA) database (accession no. DRA010021). An analysis of the GST1-1 and GST1-2 genome sequences (accession nos. LC536040 and LC536041) revealed these two alleles comprise 1623 and 1667 bases from the start to stop codons, respectively. A phylogenetic analysis indicated that GST1 belongs to the Phi-type GST group (Fig. 1a). The sequence identity between the deduced GST1 and Phi-type GSTs related to anthocyanin biosynthesis ranged from 53 to 66\%: RAP (66\%), VvGST4 (66\%), AN9 (65\%), DcGSTF2 (62\%), MdGSTF6 (66\%), CkmGST3 (68\%), and TT19 (53\%). Additionally, conserved amino acid residues specific to anthocyaninrelated GSTs were detected in GST1 (Fig. 1b).

\section{Production of GST1 genome-edited gentian lines}

The GST1 genomic sequence includes three exons and two introns. We targeted two sites on the third exon of GST1 (Fig. 2a) for genome editing with the CRISPR/ Cas9 system. By applying Agrobacterium tumefaciensmediated transformation, we obtained 54 bialaphosresistant transgenic plants and confirmed they were correctly transformed by the genomic PCR amplification of a partial Cas 9 sequence. These lines were prescreened by analyzing the sequences of PCR amplicons including target sites. Consequently, eight lines were selected, and after subcloning, Sanger sequence analyses were performed. The results indicated that seven lines were genome-edited lines in which the targeted GST1 sites were mutated (Additional file 1: Table S1). All seven of the GST1 genome-edited lines were acclimated and grown in a closed greenhouse until flowering.

\section{Next-generation sequencing (NGS) analysis of GST1 genome-edited gentian plants}

The plants bloomed at approximately 4 months after they were first acclimated. Among the seven GST1 genome-edited lines, changes to the flower color phenotypes were observed in five lines (\#3, \#12, \#13, \#23, and $\# 29)$. Line \#1 produced flowers that were similar to wild-type (WT) flowers regarding color and line \#8 did not bloom. Therefore, the amplicon sequences of the target sites in the petal transcripts and genomic DNA of these five lines were analyzed with an NGS platform. Amplicon fragment sequences were merged based on the duplicating sequence region between high-quality filtered paired-end reads. We obtained fragments constructed from $70.5-99.9 \%$ of the read pairs in each library derived from the transcripts and genomic DNA of WT and GST1 genome-edited lines. After non-specific or low-copy fragments thought to be derived from PCR and/ or sequencing errors were removed, the major fragments of each allele were counted. The proportion of major fragments in each library ranged from 86.9 to $90.2 \%$. The two alleles were distinguished based on the SNPs in the sequenced amplicon region (Fig. 2b). The sequencing results for target sites 1 and 2 in genomic DNA and the transcripts of GST1 genome-edited lines \#3 and \#12 are summarized in Table 1, with data for the other lines presented in Additional file 1: Table S2 and Additional file 3. The number of DNA fragments between alleles was almost equal and/or less than 2-fold different. Regarding the transcript analysis, there were more allele 1 fragments than allele 2 fragments in all plants. In lines \#3 and \#29, no DNA and transcript fragments corresponding to allele 1 were detected. The mutations almost corresponded with the results of the Sanger sequencing analyses presented in Additional file 1: Table S1. Amplicon sequencing analyses of the GST1 genome-edited lines uncovered several characteristic mutations, such as the deletions of $2,7,9$, and 19 bases in target sites 1 and/or 2, or more than 100 bases between target sites 1 and 2 (Table 1, Additional file 1 : Table S2). Lines \#12, \#13, and \#23 were confirmed to have heterozygous biallelic mutations. In line \#3, nine nucleotides in target site 1 were deleted, likely resulting in the deletion of three amino acids in the encoded protein. We detected a consistent mutation pattern between the genomic DNA and transcripts in all GST1 genome-edited lines.

\section{Petal phenotypes of genome-edited gentian plants}

Typical flowers of GST1 genome-edited lines are presented in Fig. 3a and Additional file 2: Figure S1A. The flowers of lines \#12, \#13, \#23, and \#29 were almost completely white. In contrast, the line \#3 flowers were similar to the WT flowers, but they were paler. The colorimetric values of the adaxial side of the flower limb area for GST1 genome-edited lines \#3 and \#12 are summarized in Fig. 3b. The colorimetric traits of the adaxial surface of petals were analyzed with a spectrophotometer and evaluated based on CIE $L^{*} a^{*} b^{*}$. To describe the color tone, the hue angle and chroma were calculated based on the recorded data $\left(a^{*}\right.$ and $\left.b^{*}\right)$. The $L^{*}, a^{*}, b^{*}$, chroma, and hue angle values of line \#12 were significantly higher and/or lower than those of the WT plants. The $L^{*}, a^{*}, b^{*}$, and chroma values of line \#3 were also significantly different from those of the WT plants, but there was no significant difference in the hue angle. These results confirmed the visible differences between the WT control and GST1 genome-edited lines. The corresponding values of the other lines are summarized in Additional file 2: Figure S1B. The colorimetric values of lines \#13, \#23, and \#29 were similar to those of line $\# 12$, but differed from those of line \#3.

\section{Analysis of anthocyanin compositions in genome-edited gentian plants}

To investigate whether the accumulated pigments in the petals of GST1 genome-edited lines changed, anthocyanin extracts were analyzed by HPLC at a monitoring 
A

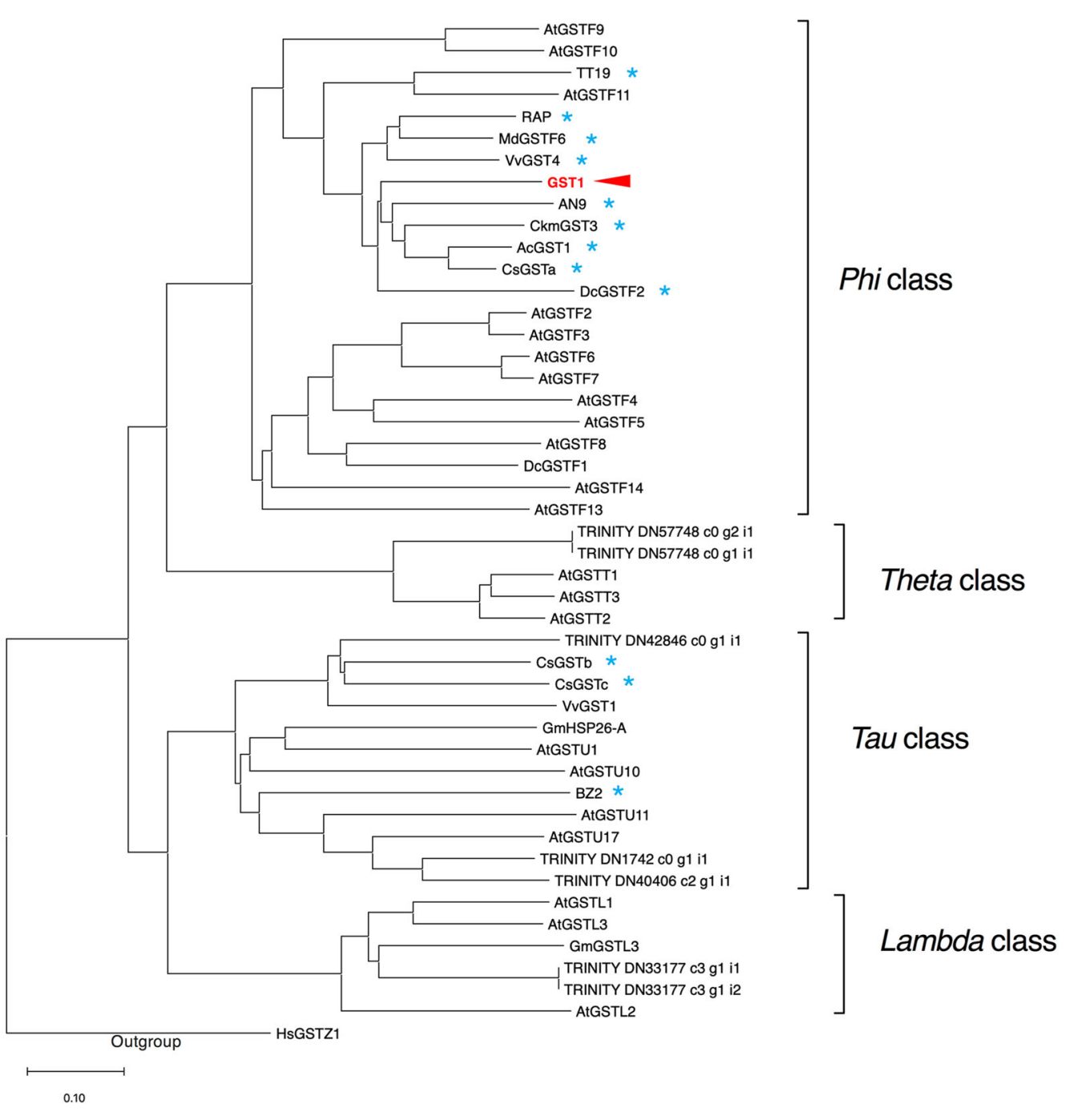

B

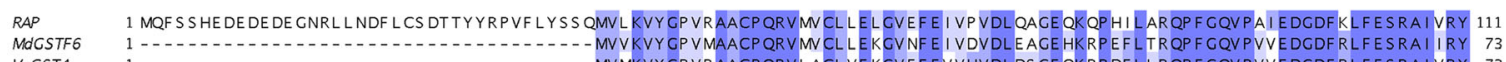
WGST4 1 T

ANG

CSTI

CKMGST3

ACGST

RAP

MACSTF

WUGST

DCCSTF2

1

1 -

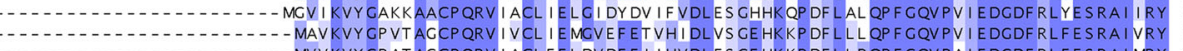

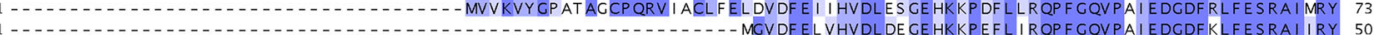

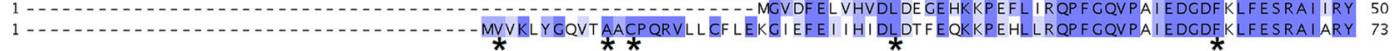
112 YAAKYAER GPN - - - LLCTTLEEKALVDQWL EVES HNF NDLVF TVVLLLVILPS MCQP CDLALVRS CEEKLKKVF DVYEERLSKSTYLACNYFSLADLSHLPAIRFLVVDEFK 219

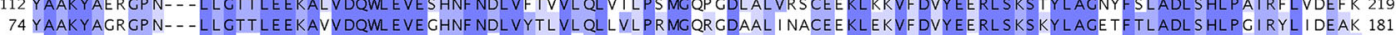
74 A AKY 74 YAAKYEVKCSK- TO

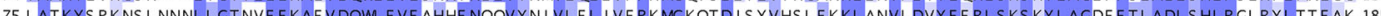
74 YA KY YOKGP 74 YAAKYADKCP CKMGST3 74 YAAKYDKGPN ACGSTI 7 Y YATKFADGN

TRP

RAP 220 MCHL ITERKNVNAWWKDISNRPAWKKLMKLAQY

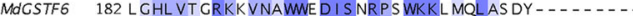

WUGST4 182 MAHLVTERKS VSAUWEDIS NRAAWKKVME AA- - -

ANG 182 FSHLVTKRKCLHEWYLDISSRDSWKKVLDL MMKKISEIEAVSIPAKEEAKV

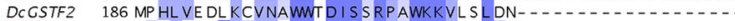

CST1 182 VCDL I KERKS VNS WWAD IS SRPAWKKVLDL MKK

CKMCST3 182 LCHMVRNRKNVNS UWMDI IS SRPS WKKVRKL MD- -

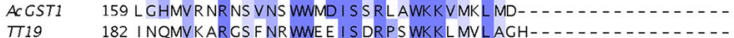

Fig. 1 (See legend on next page.) 
(See figure on previous page.)

Fig. 1 Phylogenetic tree and alignment of GSTs. a A phylogenetic tree comprising GSTs was constructed based on amino acid sequences according to the neighbor-joining method (1000 bootstrap replicates) with the CLUSTALW (Thompson et al. 1994) and MEGA programs. Blue asterisks indicate anthocyanin-related GSTs. b Alignment of anthocyanin-related GSTs with CLUSTALW and Jalview (version 2.10.3). Asterisks indicate the positions of conserved amino acid residues specific to anthocyanin-related GSTs. The red box indicates the three deleted amino acid residues due to the deletion in allele 1 of GST1 genome-edited line \#3. The accession numbers of GSTs used for constructing the phylogenetic tree and the sequence alignment are listed in Additional file 1: Table S4

wavelength of $530 \mathrm{~nm}$. The chromatograms of GST1 genome-edited lines \#3 and \#12 and the WT control are presented in Fig. 4a. The chromatogram of the WT plants included a single peak representing gentiodelphin, whereas the chromatograms of GST1 genome-edited lines \#12 and \#3 had an extremely small (ca. 6.1\% of the WT) and an intermediate-sized (ca. 63.4\% of the WT) corresponding peak, respectively. Additionally, there were no obvious peaks that were detected for lines \#3 or \#12 that were absent from the WT chromatogram. The total anthocyanin contents in the flower petals of the genome-edited and WT gentian plants were estimated by spectrophotometry (Fig. 4b). Consistent with the HPLC analysis, the total anthocyanin contents were lower in the GST1 genome-edited lines than in the WT control, with the anthocyanin levels in lines $\# 3$ and $\# 12$ approximately 71 and $<10 \%$ of that of the WT plants, respectively.

\section{Expression analysis of flavonoid biosynthesis-related genes}

Quantitative reverse transcription PCR (qRT-PCR) assays were completed to investigate the expression of GST1 and flavonoid biosynthesis-related genes during petal development. We selected two biosynthesis genes ( $C H S$ and $C H I$ ) as the early genes and four biosynthesis genes $\left(F 3^{\prime} 5^{\prime} H, D F R, A N S\right.$, and $\left.5 / 3^{\prime} A T\right)$ as the late genes. The MYB3 transcription factor gene, which regulates the expression of the late biosynthesis genes, was also included in the expression analysis. The resulting data for four developmental stages (S1-S4) (Fig. 5) revealed that the expression pattern of GST1 in WT plants was similar to those of the early genes, $\mathrm{CHS}$ and $\mathrm{CHI}$. Moreover, the GST1 expression levels were significantly lower in GST1 genome-edited lines \#3 and \#12 (ca. $32.2-63.5 \%$ of the WT) than in the WT control. There were no major differences in the expression levels and patterns of the early

\section{A}

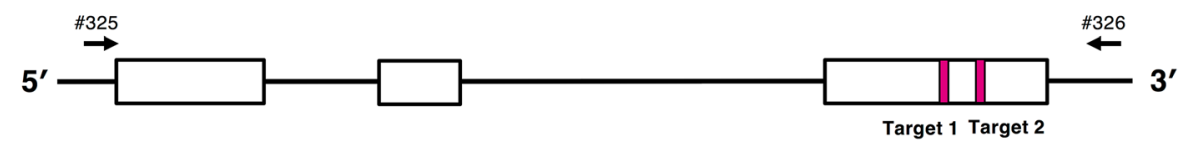

B

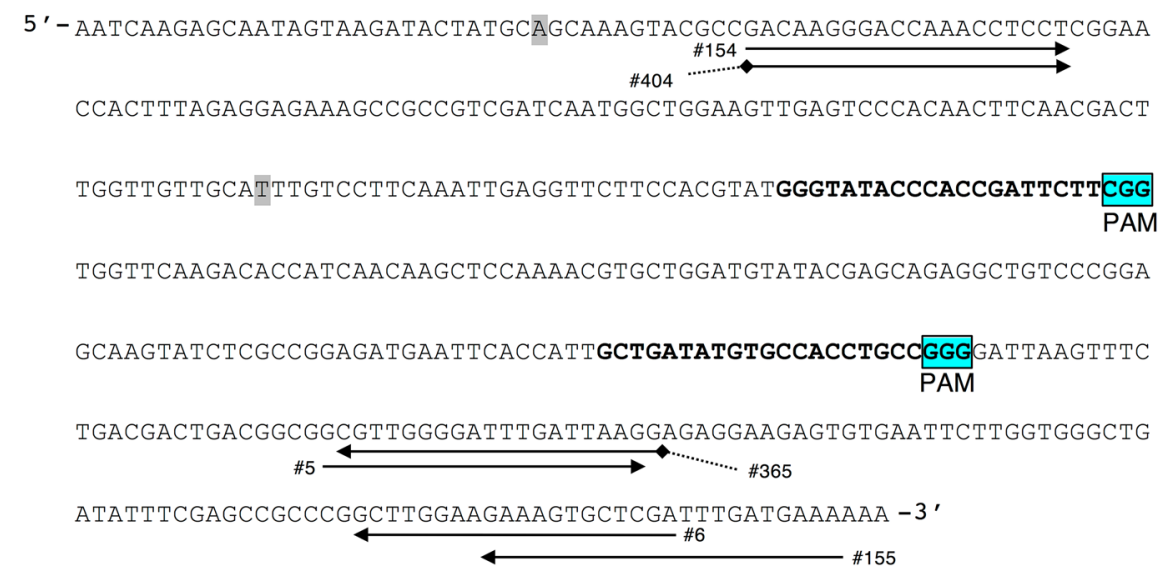

Fig. 2 Schematic diagram of GtGST1. a Schematic diagram of the GST1 genomic structure. White boxes indicate the exons. Arrows indicate the primer positions. Details regarding the primers are listed in Additional file 1: Table S3. Shaded red boxes represent target sites 1 and 2 for the genome editing with the CRISPR/Cas9 system. b Third exon sequence containing target sites 1 and 2 for genome editing. Shaded single nucleotides are the SNPs between two alleles. Bold nucleotides represent the sequences of target sites 1 and 2, and the adjacent blue shaded boxes (PAM) indicate the protospacer adjacent motif (NGG). Arrows indicate the primer positions 
Table 1 NGS analysis of PCR amplicons of DNA and RNA of GST1 genome-edited lines

\begin{tabular}{|c|c|c|c|c|c|c|c|c|c|}
\hline \multirow{2}{*}{ Line no. } & \multirow{2}{*}{ Allele } & \multicolumn{2}{|c|}{ DNA amplicons } & \multicolumn{2}{|c|}{ RNA amplicons } & \multicolumn{2}{|l|}{ Target 1} & \multicolumn{2}{|l|}{ Target 2} \\
\hline & & $\begin{array}{l}\text { Number of } \\
\text { fragments }\end{array}$ & $\begin{array}{c}\text { Rate per } \\
\text { total fragments }\end{array}$ & $\begin{array}{l}\text { Number of } \\
\text { fragments }\end{array}$ & $\begin{array}{c}\text { Rate per } \\
\text { total fragments }\end{array}$ & Sequence & In/Del & Sequence & In/Del \\
\hline \multirow{2}{*}{ WT } & 1 & 9,782 & \multirow{2}{*}{$\begin{array}{c}86.9 \% \\
(19,981 / 22,997)\end{array}$} & 20,438 & \multirow{2}{*}{$\begin{array}{c}87.7 \% \\
(24,430 / 27,851)\end{array}$} & GGGTATACCCACCGATTCTTCGG & 0 & GCTGATATGTGCCACCTGCCGGG & 0 \\
\hline & 2 & 10,199 & & 3,992 & & GGGTATACCCACCGATTCTTCGG & 0 & GCTGATATGTGCCACCTGCCGGG & 0 \\
\hline \multirow{2}{*}{ \#3 } & 1 & - & \multirow{2}{*}{$\begin{array}{c}87.9 \% \\
(25,763 / 29,295)\end{array}$} & - & \multirow{2}{*}{$\begin{array}{c}87.9 \% \\
(31,764 / 36,130)\end{array}$} & \multicolumn{3}{|c|}{ Not merged } & - \\
\hline & 2 & 25,763 & & 31,764 & & GGGTATAC----------CTTCGG & -9 & GCTGATATGTGCCACCTGCCGGG & 0 \\
\hline \multirow{2}{*}{$\# 12$} & 1 & 27,408 & \multirow{2}{*}{$\begin{array}{c}87.8 \% \\
(53,437 / 60,834)\end{array}$} & 17,831 & \multirow{2}{*}{$\begin{array}{c}88.0 \% \\
(21,053 / 23,913)\end{array}$} & -------------------TCGG & -19 & GCTGATATGT--------GCCGGG & -7 \\
\hline & 2 & 26,029 & & 3,222 & & GGGTATACCCACCGA--CTTCGG & -2 & GCTGATATGTGCCAC--GCCGGG & -2 \\
\hline
\end{tabular}

\section{A}

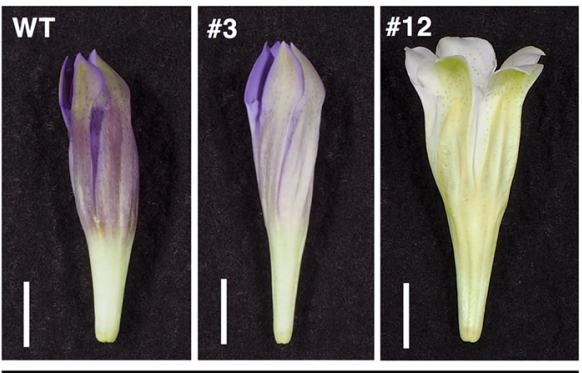

Abaxial site

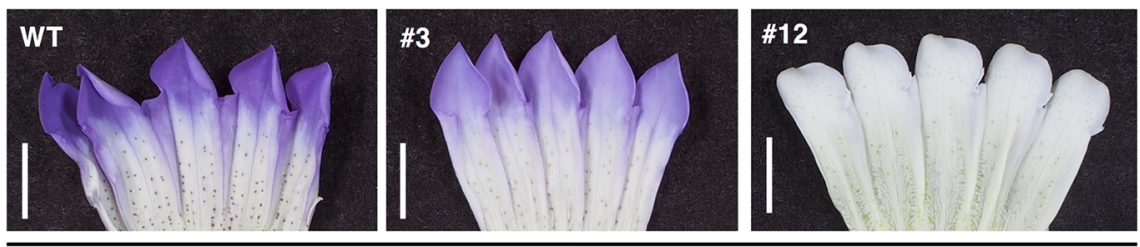

Adaxial site

B

\begin{tabular}{lcccccc}
\hline Line no. & $\begin{array}{l}\text { No. of } \\
\text { samples }\end{array}$ & $L^{*}$ & $\boldsymbol{a}^{*}$ & $\boldsymbol{b}^{*}$ & Chroma & Hue $\left(\boldsymbol{h}^{\circ}\right)$ \\
\hline \hline WT & $\mathbf{4}$ & $40.15^{\mathrm{a}^{*}}$ & $15.84^{\mathrm{a}}$ & $-49.09^{\mathrm{a}}$ & $51.59^{\mathrm{a}}$ & $287.86^{\mathrm{a}}$ \\
$\# 3$ & $\mathbf{5}$ & $47.14^{\mathrm{b}}$ & $9.10^{\mathrm{b}}$ & $-40.84^{\mathrm{b}}$ & $41.86^{\mathrm{b}}$ & $282.55^{\mathrm{a}}$ \\
$\# 12$ & 7 & $82.07^{\mathrm{c}}$ & $-4.72^{\mathrm{c}}$ & $1.41^{\mathrm{c}}$ & $4.97^{\mathrm{c}}$ & $163.50^{\mathrm{b}}$ \\
\hline
\end{tabular}

"Different letters indicate significant differences at $P<0.01$ by Tukey's HSD test.

Fig. 3 Flower color characteristics in GST1 genome-edited gentian lines. a Flower phenotypes of the wild-type control and GST1 genome-edited lines \#3 and \#12. Bar $=1 \mathrm{~cm} . \mathbf{b} L^{*}, a^{*}$, and $b^{*}$ color values at the surface of fresh petals were measured with the CM-3600A spectrophotometer (Konica Minolta, Tokyo, Japan). The chroma values and hue angles were also calculated 
A
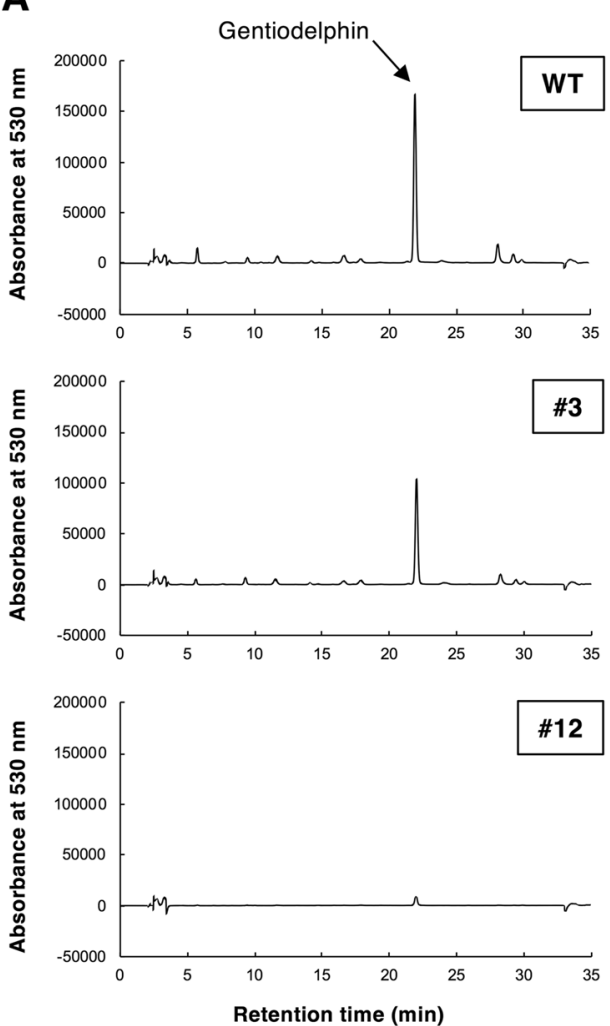

B

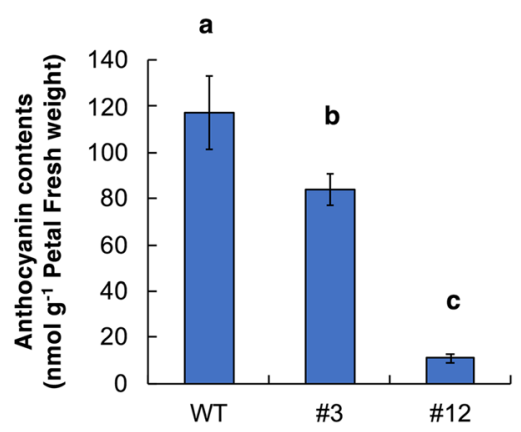

Fig. 4 Analysis of anthocyanin accumulation in the petals of GST1 genome-edited gentian lines. a Chromatogram at 530 nm for the anthocyanins in petal extracts from WT plants and GST1 genome-edited lines. b Total anthocyanin contents in the flower petals of GST1 genome-edited lines $\# 3$ and \#12 and the WT control. Quantities were estimated based on the molar absorptivity of delphinidin [ $\varepsilon$ mol = 506,783 at 530 nm, evaluated in an $80 \%$ methanol solution containing $0.1 \%(\mathrm{v} / \mathrm{v})$ trifluoroacetic acid]. Error bars represent the standard error of the means of four petals. Different letters indicate statistically significant differences according to Tukey's HSD test $(P<0.01)$

genes (CHS and $C H I)$, late genes (F3'5'H, DFR, ANS, and $5 / 3^{\prime} A T$ ), and MYB3 between the GST1 genome-edited lines and the WT plants.

\section{Complementation of the edited GST1 by particle bombardment}

To confirm whether the white coloration was due to the GST1 deficiency caused by genome editing, we performed a complementation assay involving transient GST1 expression via particle bombardment. The adaxial side of the petals of GST1 genome-edited line \#12 were bombarded with the p35S-GST1-1 (or p35S-GST1-2) and $\mathrm{p} 35 \mathrm{~S}$-sGFP recombinant plasmids just before anthesis. About $24 \mathrm{~h}$ after the bombardment, blue cells reflecting the recovery of anthocyanin accumulation were observed against a white background in some epidermal samples examined with a light microscope (Fig. 6a and c). The GFP fluorescence was also observed in the same cells under UV light (Fig. $6 \mathrm{~b}$ and d). A bombardment with p35S-GUS and p35S-sGFP did not produce blue cells, and only GFP florescence was detected (Fig. 6e and f). These results indicated that the anthocyanin accumulation of GST1 genome-edited line \#12 was restored by the transient expression of GST1.

\section{Induction of anthocyanin pigmentation in leaves}

Anthocyanin production is induced by several factors, including low temperatures, high intensity light, osmotic stress, and pathogen infections. To investigate whether GST1 genome-edited lines can accumulate anthocyanins in response to stress, we cultured them in a sugar-rich medium that usually induces anthocyanin accumulation. The WT gentian plants turned red because of the accumulation of cyanidin 3-glucoside as the major anthocyanin pigment (Fig. 7a and b). However, GST1 genome-edited line \#12 plants did not turn red and relatively little cyanidin 3-glucoside accumulated. The line \#3 plants, which produced pale blue flowers, exhibited a red coloration that was similar to that of WT plants, and there were no significant differences in the accumulation of cyanidin 3glucoside between line \#3 and WT plants (Fig. 7c). 


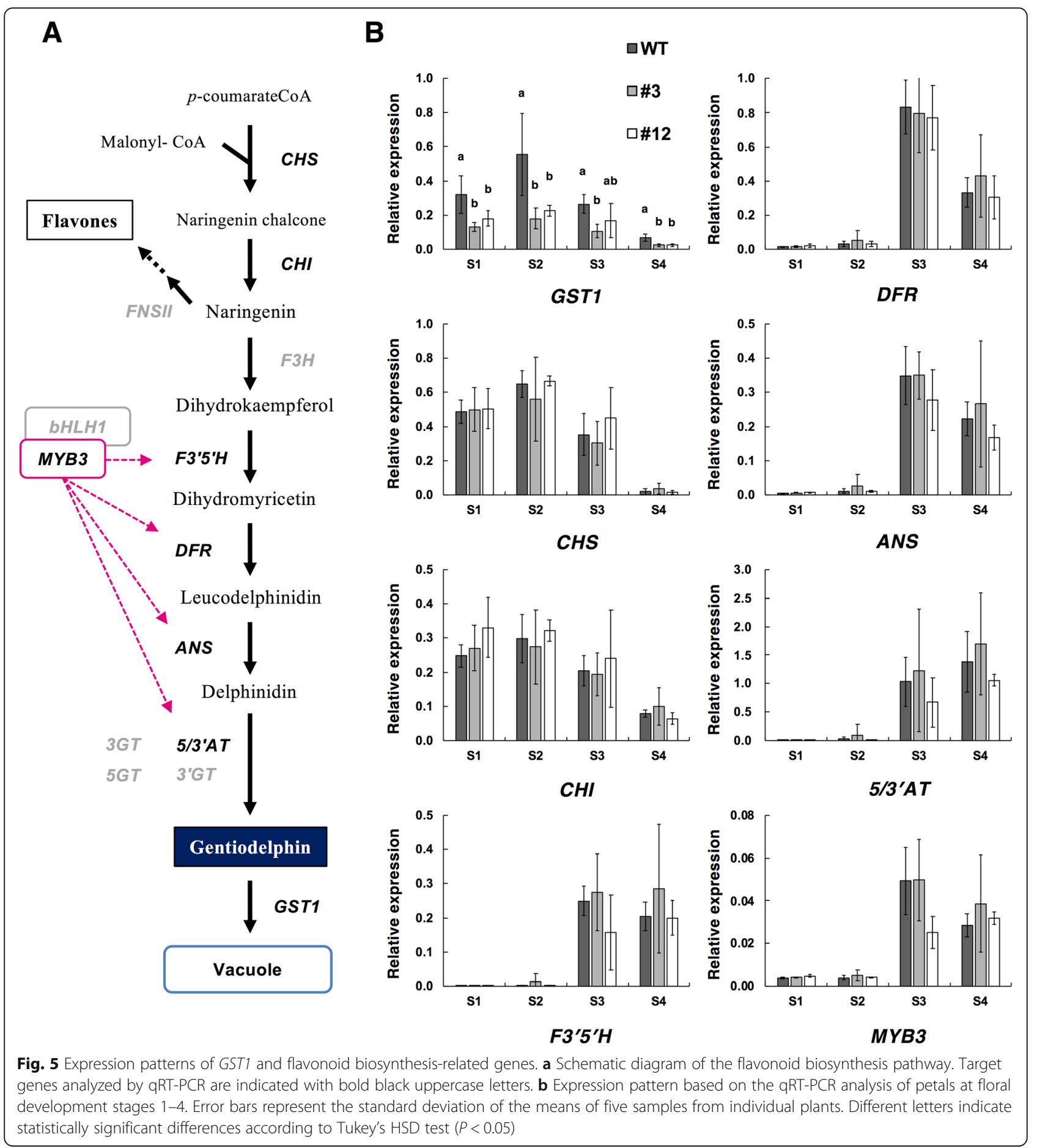

\section{Discussion}

Anthocyanins accumulate in various plant organs such as petals, fruits, seed coats, stems, leaves, and roots. These compounds are flavonoids and their contribution to flower colors has been thoroughly reviewed [23]. Additionally, their beneficial effects on human health have also been studied [24]. The flavonoid biosynthesis pathway has been well characterized in higher plants, and includes biosynthesis enzymes (e.g., chalcone synthase to anthocyanin synthase) as well as anthocyanin modification enzymes that catalyze glycosylations and acylations (Fig. 5a). Recent studies revealed that a structural-functional complex called a metabolon is constructed from flavonoid biosynthesis-related enzymes at the endoplasmic reticulum (ER) for the efficient biosynthesis of flavonoid compounds [25]. The transcriptional 


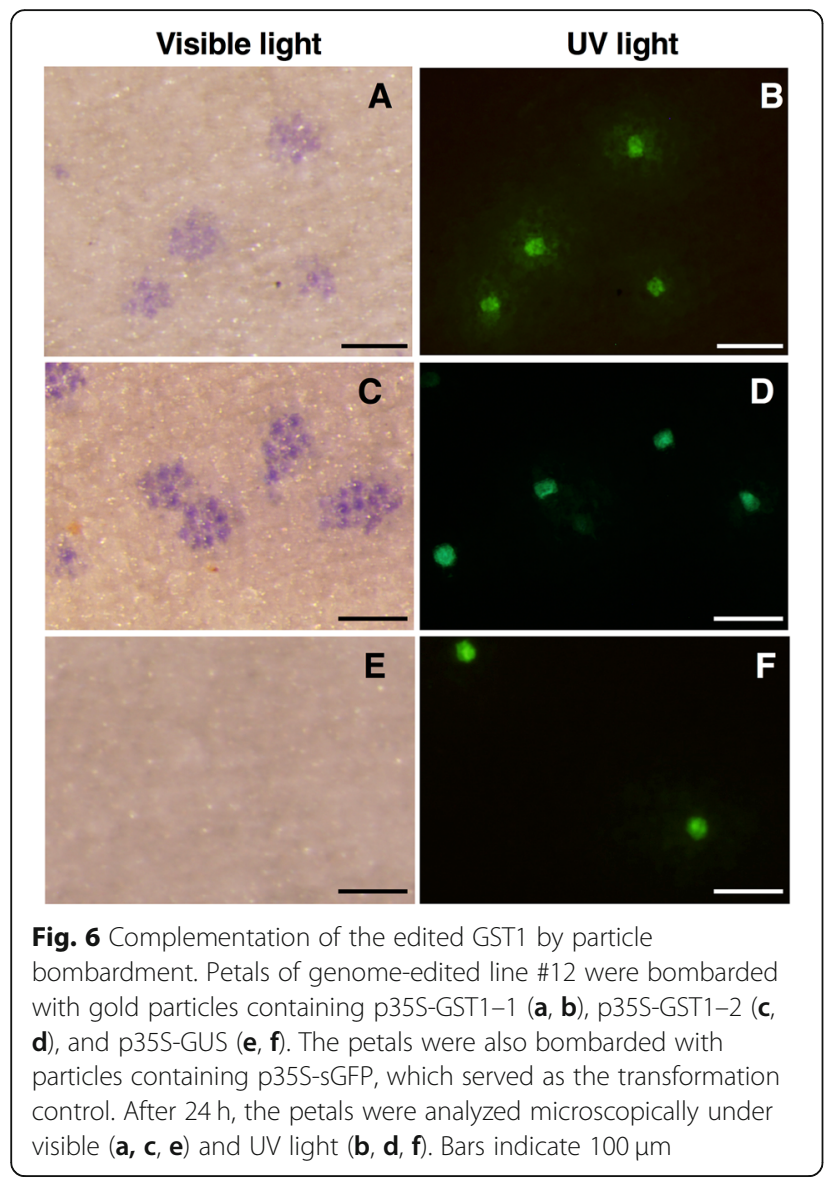

regulation of these biosynthesis genes via transcription factors, including, MYB, bHLH, and WD40, has been elucidated in many plant species [26]. As mentioned above, the biosynthesis genes and several transcription factor genes involved in gentiodelphin biosynthesis have been characterized in Japanese gentian.

Because of their water solubility, anthocyanins accumulate in vacuoles, which are membrane-bound cellular organelles present in all plants. It has been suggested that the transport of anthocyanins to vacuoles occurs via multidrug resistance-associated $A B C$ transporters [27] in a pathway that requires the conjugation of glutathione (GSH) to anthocyanins by GSTs. However, the mechanisms underlying the involvement of GSTs in anthocyanin transport remain unknown because GSH-conjugated anthocyanins have not been clearly confirmed in most cases. The Phi clade GSTs, such as AN9 (petunia) [28], AcGST1 (kiwifruit) [14], and CsGSTa (tea) [15], reportedly bind to several flavonoids. Thus, these GSTs may function as cytoplasmic flavonoid carrier proteins to transfer anthocyanins from the ER to the tonoplast.

In this study, we selected a candidate Phi-type anthocyanin-related GST gene from a draft transcriptome constructed by de novo assembly based on RNA-seq data for Gentiana triflora petals and leaves (DRA010021; BioProject PRJDB9616). On the basis of this contig sequence, GST1 was isolated from the 'Albireo' cultivar (Gentiana triflora $\times$ Gentiana scabra) (i.e., WT) and functionally analyzed. Specifically, we targeted GST1 with the CRISPR/ Cas9 genome editing system, which has been used to functionally characterize gentian genes [22]. The biallelic GST1 mutation efficiency was calculated as $13.0 \%(7 / 54$ lines) based on the Sanger sequencing and NGS amplicon sequencing data (Additional file 1: Tables S1 and S2). This efficiency was slightly better than those of previous studies regarding the gentian anthocyanin modification genes Gt5GT (5.7\%), Gt3'GT (4.9\%), and Gt5/3'AT (8.6\%) [22]. The diversity in the efficiencies was considered to be dependent on the target sequences. Of the five lines with flower colors that varied from the WT flowers, we focused on two typical GST1 knockout lines, \#3 and \#12, based on the extent of the flower color change.

The phenotypic change in line \#3 was relatively mild, with flowers that were slightly paler than the blue WT gentian flowers. During the analysis of the NGS amplicon of the target site region in genomic DNA and transcripts, the allele 1 fragment of line \#3 was not constructed with a fastq-join script because of the insertion of a specific long sequence derived from the chloroplast genome after a sequence deletion between target sites 1 and 2 (data not shown). Thus, we considered that this mutation eliminated the translation of a functional protein from allele 1. Additionally, we speculated that the deletion in allele 2 of line \#3 was not a crucial mutation because it resulted in the deletion of only three amino acids without a frameshift. As indicated in Fig. 3a, we observed a mild flower color change in line \#3 and there was no substantial decrease in the anthocyanin content compared with the WT level (Fig. 4b). Furthermore, there were no differences in the hue angles at the petal surface between line \#3 and WT plants (Fig. 3b). The peak patterns in the petal extract HPLC chromatograms were also the same between line \#3 and WT plants, with the same minor peaks detected (Fig. 4a). These results suggested that the deletion at target site 1 of GST1 exon 3 , which eliminated three amino acids, resulted in a functional protein that was slightly less active than normal. A consequence of this decreased activity was the slight inhibition of gentiodelphin accumulation that caused the mild flower color change (i.e., GST1 knockdown).

In contrast, in GST1 genome-edited line \#12, the flowers were faint blue to nearly white. Moreover, the total anthocyanin content in line \#12 was less than $10 \%$ of that of the WT control. The biallelic genome editing was confirmed for both target sites 1 and 2 (Table 1 ). The mutations were considered to cause frameshifts and unusual stop codons. Other GST1 genome-edited lines 
A
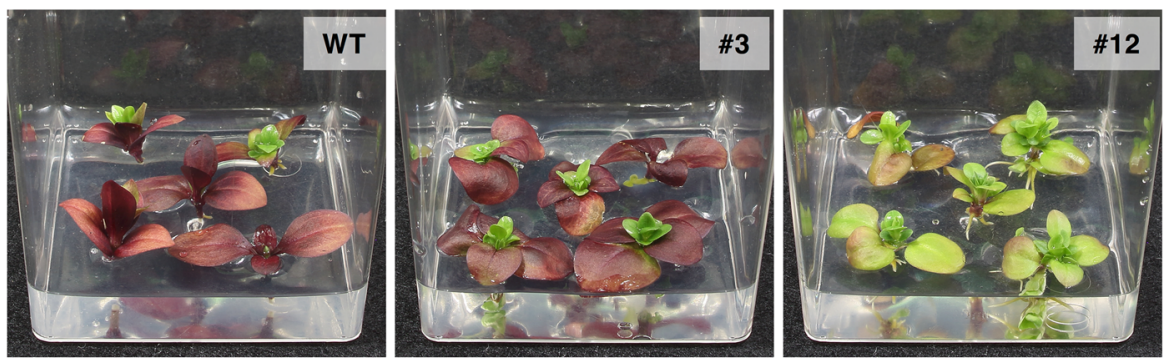

B

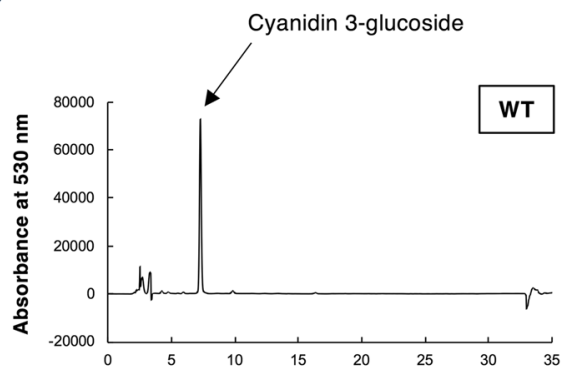

C
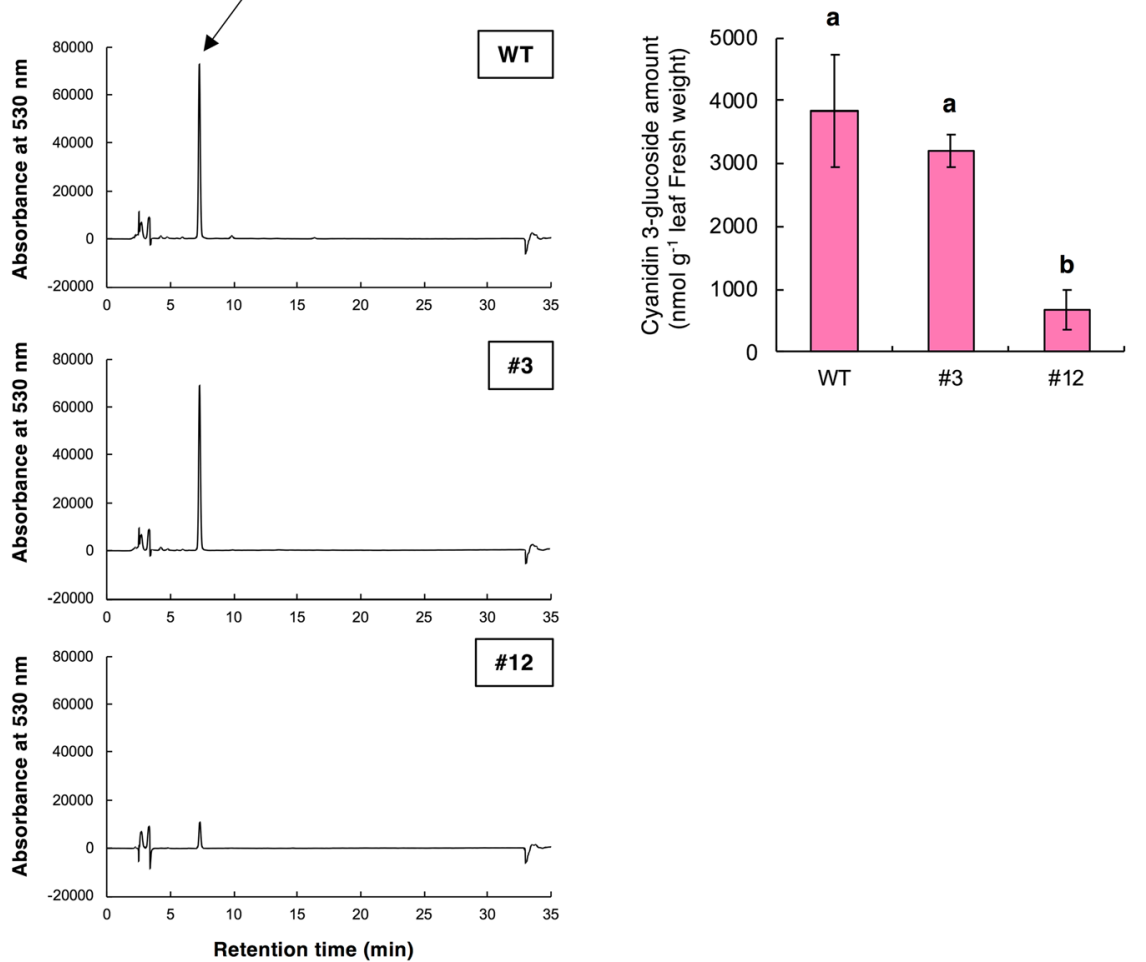

Fig. 7 Induction of anthocyanin accumulation in the leaves of GST1 genome-edited gentian lines. a Appearance of WT and GST1 genome-edited gentian plants on half-strength MS medium supplemented with 10\% sucrose at 43 days after being transferred. $\mathbf{b}$ HPLC chromatograms at $365 \mathrm{~nm}$ of leaf extracts from plants grown on half-strength MS medium supplemented with $10 \%$ sucrose at 43 days after being transferred. The main peak is cyanidin 3-glucoside. c Anthocyanin contents in the leaves of the WT control and GST1 genome-edited lines. Quantities were determined based on the standard curve generated from the HPLC peak area of six serial dilutions of cyanidin 3-glucoside. Error bars represent the standard error of the means of five leaves from individual plants. Different letters indicate statistically significant differences according to Tukey's HSD test $(P<0.01)$

(i.e., \#13, \#23, and \#29), which were also confirmed to have biallelic mutations at the target sites (Additional file 1: Table S1), produced flower colors similar to those of line \#12 (Additional file 2: Fig. S1). Thus, we concluded the genome editing of GST1 was successful, with lines \#12, \#13, \#23, and \#29 exhibiting a gene knockout phenotype in contrast to the gene knockdown phenotype of line \#3.

Because it was unclear whether the observed phenotypes were due to GST1 deficiency, we performed a complementation assay. The blue coloration of petals was restored in GST1 genome-edited line \#12 by the transient expression of either GST1-1 or GST1-2 (Fig. 6), indicating the white-flowered phenotype was indeed caused by a GST1 mutation. The importance of GST1 was also demonstrated. Specifically, even if the anthocyanin biosynthesis pathway is active, the final transport mediated by GST1 is necessary for the accumulation of anthocyanins in gentian plants.

The qRT-PCR data for developing flowers revealed similar expression patterns among GST1, CHS, and CHI, 
with early expression that peaked at stage 2 . In this stage, GST1 expression levels were significantly lower in both GST1 genome-edited lines than in the WT plants. This may have been related to nonsense-mediated mRNA decay (i.e., eukaryotic mRNA quality control) $[29,30]$. Transcription factors that regulate the expression of anthocyanin-related GST genes have been identified in kiwifruit (AcMYBF110 for AcGST1) [14] and apple (MdMYB1 for MdGSTF6) [16]. On the basis of the gentian GST1 expression pattern (Fig. 5b), it is unlikely that GtMYB3 regulates GST1 expression. The downregulated expression of GST1 did not significantly affect the expression levels of the examined flavonoid-biosynthetic genes, suggesting feedback regulation via GST did not influence anthocyanin accumulation. Unfortunately, we did not identify the transcription factor regulating GST1 expression in gentian plants. Therefore, additional studies are necessary to clarify GST1 transcriptional regulation.

Anthocyanin accumulation is induced by an exogenous sugar treatment, and the regulatory genes involved in the associated sucrose signaling have been characterized [31, 32]. In this study, we observed that the sugar-induced anthocyanin pigmentation in in vitro cultured plants was inhibited in GST1 genome-edited lines (Fig. 7). This inhibition was severe in knockout line \#12, which was consistent with the flower color phenotype. These results implied that GST1 also contributes to the stress-induced anthocyanin accumulation in gentian plants, although more detailed analyses of GST expression in other organs are necessary. From a practical perspective, leaf pigmentation is usually an undesirable trait for ornamental flowers. Therefore, the mechanism regulating stress-induced anthocyanin accumulation via anthocyanin transport should be elucidated.

Our GST1 genome-edited gentian lines did not produce completely white flowers, implying other proteins are also involved in the anthocyanin transport of gentian plants. We selected and analyzed a Phi (F) class GST in this study. However, several genes encoding Tau (U) class GSTs, such as Bz2 in Zea mays [33] and VviGST1 in Vitis vinifera [34], are also associated with anthocyanins. More recently, CsGSTb and CsGSTc from Camellia sinensis [15] were confirmed as Tau clade GST genes related to anthocyanin transport. Our phylogenetic analysis (Fig. 1a) indicated that a GST gene, TRINITY DN42846_c0_g1_i1, which was detected based on the de novo gentian transcripts, belongs in the Tau clade, with deduced amino acid sequence identities of 42, 54, and 55\% with VviGST1, CsGSTb, and CsGSTc, respectively. Thus, future studies should examine Tau-type GSTs as well as the $\mathrm{ABC}$ transporters to elucidate the mechanism underlying anthocyanin accumulation in gentian plants.

\section{Conclusions}

In this study, we isolated and analyzed an anthocyaninrelated GST gene, GST1, in cultivated Japanese gentian. Its contribution to flower pigmentation was confirmed with the CRISPR/Cas9 genome editing system. We produced several GST1 genome-edited lines and analyzed the effects of their mutations on flower color. The data presented herein revealed two distinct phenotypes, namely knockout (almost white) and knockdown (pale blue) phenotypes. These results suggest genome editing with the CRISPR/Cas9 system can be applied to knock out target genes as well as slightly modify an allele of a gene of interest. Our study findings may be relevant for clarifying the anthocyanin transport in higher plants. Moreover, our mutant lines may be useful for gentian breeding because such mutations have not been detected in natural mutants. Our results indicate that GST1 helps mediate the anthocyanin accumulation in gentian plants, although additional related genes remain to be identified. The use of genome editing technology will enhance future studies of anthocyanin transport in gentian plants.

\section{Methods}

Plant materials and the selection of transformants

Blue-flowered gentian cultivar 'Albireo' (Gentiana triflora $\times$ Gentiana scabra) (i.e., WT) was used for the targeted genome editing with the CRISPR/Cas9 system. This cultivar, which was identified by Dr. Masahiro Nishihara, was bred in Iwate prefecture and is registered in the Plant Variety Protection database at Ministry of Agriculture, Forestry and Fisheries, Japan (registration no. 2553). It was kindly provided by the Iwate Agricultural Research Center. The WT plants were maintained in an in vitro culture using half-strength MS medium supplemented with $3 \%$ sucrose as the basal medium under the same cultivation conditions in our previous study [22].

Gentian plants were transformed according to an $A$. tumefaciens-mediated procedure, after which the genome-edited transformants were selected as previously described [22, 35]. Briefly, transformed shoots were screened on medium containing $0.75 \mathrm{mg} \mathrm{l}^{-1}$ bialaphos. Candidate transgenic plants were selected based on the PCR amplification of a pcoCas9 fragment with MightyAmp DNA polymerase (TaKaRa, Shiga, Japan). Primers $\# 61$ and \#62, which are specific for pcoCas9, were used for the PCR analysis of crude leaf extracts as the template according to the manufacturer's instructions. The genome-edited plants were selected by detecting insertions or deletions in the target sites via direct- and subcloning-sequence analyses of the PCR products amplified with primers \#154 and \#155 specific for the target site region and the above-described method (Fig. 2a). Details regarding all primers are listed in Additional file 
1: Table S3. The regenerated GST1 genome-edited lines were acclimated to the closed greenhouse or cultured in an incubation room under light-emitting diode lamps as described previously until they bloomed [36]. Petals and leaves were harvested, immediately frozen in liquid nitrogen, and stored at $-80^{\circ} \mathrm{C}$ until analyzed.

\section{Isolation of GST cDNA and genomic sequences related to anthocyanin accumulation}

Single strand cDNAs were synthesized from the total RNA extracted from the petals of WT plants and used for PCR amplification as described previously [22]. The sequences used for designing primers specific for gentian GST genes were obtained from the DDBJ DRA database (accession no. DRA010021). From more than 130,000 contigs, 30 contigs were identified as GST genes based on BLASTX (version 2.2.26) matches, including one that was annotated with the GO term "anthocyanin-containing compound metabolic process". We designed primers \#276 and \#277 based on the sequencing data for this GST contig for the subsequent 3'-rapid amplification of cDNA ends with the GeneRacer kit (Invitrogen, USA). The full-length GST1 sequence was amplified by PCR with primers \#325 and \#326, after which the amplicon was sequenced with the Big-Dye Terminator Cycle Sequencing kit (version 1.1) and the ABI PRISM 3130xl or 3500 Genetic Analyzers (Applied Biosystems, Foster City, CA, USA).

To isolate the full-length GST1 genomic sequence, genomic DNA was isolated from the young leaves of WT gentian plants with the GeneElute Genomic DNA Isolation system (Sigma-Aldrich, St Louis, MO, USA). The PCR amplification with primers \#325 and \#326 and the sequencing of the amplicon were performed as described above. The primers used for isolating GST1 are listed in Additional file 1: Table S3. The GST coding regions were translated to the respective deduced amino acid sequences with ExPASy (https://web.expasy.org/ translate/). A phylogenetic tree was produced and GST amino acid sequences were aligned with the CLUSTALW (Thompson et al. 1994), MEGA (version 10.1.7), and Jalview (version 2.10.3) programs. The GST protein sequences used for constructing the phylogenetic tree are listed in Additional file 1: Table S4.

\section{Construction of a binary vector for editing GST1 in the gentian genome}

A binary CRISPR/Cas9 vector for targeting gentian GST1 was constructed to harbor two single-guide RNA expression cassettes using the same procedure in our previous study [22]. The two target sites in GST1 exon 3 are presented in Fig. 2a. The resultant binary vector pSbar-pcoCas9-AtU626p-GST1target-1gRNA-AtU6-26p-GST1target2gRNA
(Fig. 2b) was transformed into A. tumefaciens strain EHA101 by electroporation and used for gentian transformation.

\section{Next-generation sequencing analysis of the genome- edited target site region of GST1}

The genomic DNA and transcripts in the petals of the GST1 genome-edited lines and WT plants were analyzed with the Illumina MiSeq Next-generation Sequencer to confirm the target site sequences. Genomic DNAs and cDNAs were prepared as described above. The extracted RNA samples were reverse transcribed with PrimeScript II (TaKaRa). Amplicon sequencing was performed on an Illumina system as described previously [21]. Briefly, raw reads were pre-processed with the FASTX toolkit, after which a single contiguous sequence (fragment) containing the target site region was constructed with a fastq-join script [37]. Finally, all unique fragments were counted. The primers used for the NGS analysis are listed in Additional file 1: Table S1.

\section{Measurement of gentian petal colors}

Color differences were determined based on CIE $L * a * b$, in which $L^{*}$ indicates lightness, $a^{*}$ is the red/green coordinate, and $b^{*}$ is the yellow/blue coordinate. To quantify the flower color phenotypes of the GST1 genome-edited lines and WT plants, the colorimetric values $L^{*}, a^{*}$, and $b^{*}$ as well as the chroma $\left(C^{*}\right)$ value and hue angle, which were calculated based on the $L^{*}$, $a^{*}$, and $b^{*}$ values, of the adaxial surface of the limb area of fresh petals were measured. The benchtop CM-3600A spectrophotometer (Konica Minolta, Tokyo, Japan) was used for this analysis.

\section{Analysis of anthocyanin compositions in the petals of GST1 genome-edited gentian plants}

The anthocyanin compositions of petal extracts were analyzed by HPLC system [PU-4180 PUMP, MD-4010 photodiode array detector, and ChromNAV (version 2.03.05) software; JASCO, Tokyo, Japan] equipped with a Unifinepak C18 column (4.6 mm internal diameter $\times$ $250 \mathrm{~mm}$; JASCO). Anthocyanins were extracted from fresh petals and subjected to HPLC analysis as described previously [22]. We used a linear gradient elution (1.1 $\mathrm{ml} \mathrm{min-1)}$ of $14-22 \%$ acetonitrile in $1 \%$ aqueous phosphoric acid over $30 \mathrm{~min}$. The V-730BIO spectrophotometer (JASCO) was used to estimate anthocyanin concentrations based on the molar absorptivity of delphinidin chloride $[\varepsilon \mathrm{mol}=506,783$ at $530 \mathrm{~nm}$, evaluated in the $80 \%$ methanol solution containing $0.1 \%(\mathrm{v} / \mathrm{v})$ trifluoroacetic acid]. 


\section{Expression analysis of GST1 and flavonoid biosynthesis- related genes}

Total RNA was extracted from flower petals collected at four developmental stages as described above, after which cDNA was synthesized with the PrimeScript ${ }^{\mathrm{tm}} \mathrm{RT}$ reagent Kit with gDNA Eraser (Perfect Real Time) (TaKaRa) according to the manufacturer's instructions. A qRT-PCR assay was completed with TB Green ${ }^{\mathrm{nx}}$ Premix Ex Taq ${ }^{\text {Tix }}$ II (Tli RNaseH Plus) (TaKaRa) and the QuantStudio 5 Real-Time PCR system (Applied Biosystems Japan). The reaction mixtures (20 $\mu$ l total volume) consisted of the following components: $10 \mu \mathrm{l}$ master mix, $0.8 \mu \mathrm{M}$ each primer, and $1 \mu \mathrm{l} \mathrm{cDNA}$ template. The qRT-PCR primer sets for GST1, CHS, CHI, F3'5' $H$, $D F R, A N S, 5 / 3^{\prime} A T$, and $M Y B$ are listed in Additional file 1: Table S3. The PCR conditions were as follows: $50^{\circ} \mathrm{C}$ for $2 \mathrm{~min} ; 95^{\circ} \mathrm{C}$ for $10 \mathrm{~min}$; 40 cycles of $95^{\circ} \mathrm{C}$ for $15 \mathrm{~s}$ and $60^{\circ} \mathrm{C}$ for $1 \mathrm{~min}$. A melting curve analysis was performed to verify the specificity and identity of the qRT-PCR products. The expression levels of each gene were normalized against the $U B Q$ expression level and recorded as relative values based on the maximum expression levels.

\section{Complementation of the edited GST1 by particle bombardment}

In this study, p35S-sGFP, containing sGFP under the control of the cauliflower mosaic virus $35 \mathrm{~S}$ promoter, was used as the transformation control plasmid, whereas p35S-GUS was used as the negative control plasmid. The $s G F P$ fragment was replaced with a modified GST1-1 (or GST1-2) (six base substitutions in the target site; details in Additional file 2: Fig. S2) designed to disrupt the editing by the integrated CRIS PR/Cas9 construct, resulting in p35S-GST1-1 and p35S-GST1-2. The recombinant plasmids were coprecipitated onto gold particles. The PDS-1000/He Biolistic Particle Delivery System (Bio-Rad, CA) was used to bombard the GST1 genome-edited gentian petals with the particles as previously described [38]. After $24 \mathrm{~h}$, the petals were analyzed microscopically under visible and UV light.

\section{Induction of anthocyanin pigmentation in leaves}

The WT and GST1 genome-edited gentian plants maintained by in vitro cultivation were transferred to half-strength MS medium supplemented with 10\% sucrose. Plants were grown in a culture room set at $22^{\circ} \mathrm{C}$ under a 16-h light/8-h dark photoperiod, with light supplied by white-light-emitting diodes. An HPLC analysis was performed as described above. Anthocyanin contents were estimated based on the standard curve generated from the HPLC peak area of six serial dilutions of cyanidin 3-glucoside.

\section{Supplementary information}

Supplementary information accompanies this paper at https://doi.org/10. 1186/s12870-020-02565-3.

Additional file 1: Table S1. Sanger sequencing analysis of the PCR amplicons of the DNA from GST1 genome-edited lines and the WT control. Table S2. Next-generation sequencing analysis of the PCR amplicons of the DNA and RNA from GST1 genome-edited lines and the WT control. Table S3. Primers used in this study. Table S4. Glutathione S-transferase proteins used for constructing the phylogenetic tree in Fig. 1a.

Additional file 2: Figure S1. Flower color characteristics in GST1 genome-edited gentian lines \#13, \#23, and \#29. (A) Flower color phenotypes of GST1 genome-edited lines \#13, \#23, and \#29. (B) $L^{*}, a^{*}$, and $b^{*}$ color values at the surface of fresh petals were measured with the CM3600A spectrophotometer (Konica Minolta, Tokyo, Japan). The chroma values and hue angles were also calculated. Figure $\mathbf{S 2}$ Sequence of the third exon of the modified GST1 for the complementation assay. The highlighted nucleotides (i.e., shaded, bold, or boxed) are the same as those in Fig. 2b. The substituted nucleotides for the transient expression assay are indicated in red. These substitutions did not change the encoded amino acids.

Additional file $\mathbf{3}$ The results of next-generation amplicon sequencing of WT and GST1 genome-edited gentian lines. Numbers after sequence names in the Excel worksheets are sequential serial numbers and read counts of each fragment.

\section{Abbreviations}

GST: Glutathione S-transferase; CRISPR: Clustered regularly interspaced short palindromic repeats; NGS: Next-generation sequencing; PCR: Polymerase chain reaction; HPLC: High-performance liquid chromatography

\section{Acknowledgments}

We thank Dr. Holger Puchta (Botanical Institute, Karlsruhe Institute of Technology, Germany) for providing pcoCAS9. Some of the computational analyses were completed with the NIG supercomputer at the ROIS National Institute of Genetics. We also thank Drs. Nobuhiro Sasaki, Toyo University, Japan, and Keiichirou Nemoto, Iwate Biotechnology Research Center, for their helpful discussions. The authors are grateful to Mses. R. Washiashi and Y. Kurokawa, Iwate Biotechnology Research Center, for their technical support. We thank Edanz Group (https://enauthor-services.edanzgroup.com/) for editing a draft of this manuscript.

\section{Authors' contributions}

$\mathrm{KT}$ and MaN conceived and designed the experiments. AH and AW prepared and treated the test materials. KT, MY, and MiN analyzed the data. KT contributed to the NGS analysis. MaN supervised the study. KT wrote the manuscript. MaN critically read and reviewed the manuscript. All authors approved the final manuscript.

\section{Funding}

This work was financially supported by Iwate Prefecture and also in part by Grants-in-Aid for Scientific Research from the Japan Society for the Promotion of Science (16 K18654 and 18 K14462). This work was also supported in part by commissioned projects, "Breeding of floricultural plants adapted for high practical needs and development of low cost cultivation techniques" and "Development of new varieties and breeding materials in crops by genome editing", of the Ministry of Agriculture, Forestry and Fisheries, Japan. The funding agencies were not involved in designing the experiments or analyzing the data.

\section{Availability of data and materials}

The data generated during this study are included in this published article and its additional files. Primary datasets used and/or analyzed during the current study available from the corresponding author on reasonable request.

Ethics approval and consent to participate Not applicable.

Consent for publication

Not applicable. 


\section{Competing interests}

The authors declare that they have no competing interests.

\section{Author details}

'Iwate Biotechnology Research Center, 22-174-4 Narita, Kitakami, Iwate 024-0003, Japan. ${ }^{2}$ Present address: Tokyo University of Agriculture, 1737 Funako, Atsugi, Kanagawa 243-0034, Japan. ${ }^{3}$ Tokyo University of Agriculture, 1737 Funako, Atsugi, Kanagawa 243-0034, Japan.

Received: 15 April 2020 Accepted: 21 July 2020

Published online: 06 August 2020

\section{References}

1. Goto T, Kondo T, Tamura H, Imagawa H, lino A, Takeda K. Structure of gentiodelphin, an acylated anthocyanin from Gentiana maikinoi, that is stable in dilute aqueous solution. Tetrahedron Lett. 1982;23:3695-8.

2. Yoshida K, Toyama Y, Kameda K, Kondo T. Contribution of each caffeoyl residue of the pigment molecule of gentiodelphin to blue color development. Phytochemistry. 2000;54:85-92.

3. Yoshida K, Mori M, Kondo T. Blue flower color development by anthocyanins: from chemical structure to cell physiology. Nat Prod Rep. 2009:26:884-915.

4. Nakatsuka T, Haruta KS, Pitaksutheepong C, Abe Y, Kakizaki Y, Yamamoto K, Shimada N, Yamamura S, Nishihara M. Identification and characterization of R2R3-MYB and bHLH transcription factors regulating anthocyanin biosynthesis in gentian flowers. Plant Cell Physiol. 2008;49:1818-29.

5. Nakatsuka T, Saito M, Yamada E, Nishihara M. Production of picotee-type flowers in Japanese gentian by CRES-T. Plant Biotechnol. 2011;28:173-80.

6. Tasaki K, Atsumi G, Nishihara M, Sekine KT. Development of a Broad bean wilt virus 2-based expression vector for gentian. Sci Hort. 2016;201:279-86.

7. Nakatsuka T, Sasaki N, Nishihara M. Transcriptional regulators of flavonoid biosynthesis and their application to flower color modification in Japanese gentians. Plant Biotechnol. 2014;31:389-99.

8. Nakatsuka T, Saito M, Sato-Ushiku Y, Yamada E, Nakasato T, Hoshi N, Fujiwara K, Hikage T, Nishihara M. Development of DNA markers that discriminate between white- and blue-flowers in Japanese gentian plants. Euphytica. 2011:184:335-44.

9. Kakizaki Y, Nakatsuka T, Kawamura H, Abe J, Abe Y, Yamamura S, Nishihara M. Development of codominant DNA marker distinguishing pink from blue flowers in Gentiana scabra. Breed Res. 2009;11:9-14.

10. Nishihara M, Tasaki K, Sasaki N, Takahashi H. Development of basic technologies for improvement of breeding and cultivation of Japanese gentian. Breed Sci. 2018;68:14-24.

11. Zhao J. Flavonoid transport mechanisms: how to go, and with whom. Trends Plant Sci. 2015;20:576-85.

12. Sasaki N, Nishizaki $Y$, Uchida $Y$, Wakamatsu $E$, Umemoto N, Momose M, Okamura M, Yoshida H, Yamaguchi M, Nakayama M, et al. Identification of the glutathione S-transferase gene responsible for flower color intensity in carnations. Plant Biotechnol. 2012;29:223-7.

13. Kitamura S, Akita Y, Ishizaka H, Narumi I, Tanaka A. Molecular characterization of an anthocyanin-related glutathione S-transferase gene in cyclamen. J Plant Physiol. 2012;169:636-42.

14. Liu Y, Qi Y, Zhang A, Wu H, Liu Z, Ren X. Molecular cloning and functional characterization of ACGST1, an anthocyanin-related glutathione S-transferase gene in kiwifruit (Actinidia chinensis). Plant Mol Biol. 2019;100:451-65.

15. Liu Y, Jiang H, Zhao Y, Li X, Dai X, Zhuang J, Zhu M, Jiang X, Wang P, Gao L, et al. Three Camellia sinensis glutathione S-transferases are involved in the storage of anthocyanins, flavonols, and proanthocyanidins. Planta. 2019;250: 1163-75.

16. Jiang $\mathrm{S}$, Chen $\mathrm{M}, \mathrm{He} \mathrm{N}$, Chen X, Wang $\mathrm{N}$, Sun Q, Zhang T, Xu H, Fang $\mathrm{H}_{\text {, }}$ Wang $Y$, et al. MdGSTF6, activated by MdMYB1, plays an essential role in anthocyanin accumulation in apple. Hortic Res. 2019;6:40.

17. Soda N, Verma L, Giri J. CRISPR-Cas9 based plant genome editing: significance, opportunities and recent advances. Plant Physiol Biochem. 2018;131:2-11

18. Ma X, Zhang Q, Zhu Q, Liu W, Chen Y, Qiu R, Wang B, Yang Z, Li H, Lin Y, et al. A robust CRISPR/Cas9 system for convenient, high-efficiency multiplex genome editing in monocot and dicot plants. Mol Plant. 2015;8:1274-84.

19. Zhang Y, Malzahn AA, Sretenovic S, Qi Y The emerging and uncultivated potential of CRISPR technology in plant science. Nat Plants. 2019;5:778-94.
20. Watanabe K, Kobayashi A, Endo M, Sage-Ono K, Toki S, Ono M. CRISPR/Cas9mediated mutagenesis of the dihydroflavonol-4-reductase-B (DFR-B) locus in the Japanese morning glory Ipomoea (Pharbitis) nil. Sci Rep. 2017;7:10028.

21. Nishihara M, Hiquchi A, Watanabe A, Tasaki K. Application of the CRISPR/ Cas9 system for modification of flower color in Torenia fournieri. BMC Plant Biol. 2018;18:331.

22. Tasaki K, Higuchi A, Watanabe A, Sasaki N, Nishihara M. Effects of knocking out three anthocyanin modification genes on the blue pigmentation of gentian flowers. Sci Rep. 2019;9:15831.

23. Iwashina T. Contribution to flower colors of flavonoids including anthocyanins: a review. Nat Prod Commun. 2015:10:529-44.

24. Khoo HE, Azlan A, Tang ST, Lim SM. Anthocyanidins and anthocyanins: colored pigments as food, pharmaceutical ingredients, and the potential health benefits. Food Nutr Res. 2017;61:1361779.

25. Nakayama T, Takahashi S, Waki T. Formation of flavonoid Metabolons: functional significance of protein-protein interactions and impact on flavonoid Chemodiversity. Front Plant Sci. 2019;10:821.

26. $\mathrm{Xu} \mathrm{W}$, Dubos C, Lepiniec L. Transcriptional control of flavonoid biosynthesis by MYB-bHLH-WDR complexes. Trends Plant Sci. 2015;20:176-85.

27. Behrens CE, Smith KE, lancu CV, Choe JY, Dean JV. Transport of Anthocyanins and other flavonoids by the Arabidopsis ATP-binding cassette transporter AtABCC2. Sci Rep. 2019:9:437.

28. Mueller LA, Goodman CD, Silady RA, Walbot V. AN9, a petunia glutathione $S$-transferase required for anthocyanin sequestration, is a flavonoid-binding protein. Plant Physiol. 2000;123:1561-70.

29. Lindeboom RGH, Vermeulen M, Lehner B, Supek F. The impact of nonsensemediated mRNA decay on genetic disease, gene editing and cancer immunotherapy. Nat Genet. 2019:51:1645-51.

30. Tuladhar R, Yeu Y, Tyler Piazza J, Tan Z, Rene Clemenceau J, Wu X, Barrett Q, Herbert J, Mathews DH, Kim J, et al. CRISPR-Cas9-based mutagenesis frequently provokes on-target mRNA misregulation. Nat Commun. 2019;10:4056.

31. Ai TN, Naing AH, Arun M, Lim SH, Kim CK. Sucrose-induced anthocyanin accumulation in vegetative tissue of Petunia plants requires anthocyanin regulatory transcription factors. Plant Sci. 2016;252:144-50.

32. Li Y, Van den Ende W, Rolland F. Sucrose induction of anthocyanin biosynthesis is mediated by DELLA. Mol Plant. 2014;7:570-2.

33. Marrs KA, Alfenito MR, Lloyd AM, Walbot V. A glutathione S-transferase involved in vacuolar transfer encoded by the maize gene Bronze-2. Nature. 1995:375:397-400

34. Conn S, Curtin C, Bezier A, Franco C, Zhang W. Purification, molecular cloning, and characterization of glutathione S-transferases (GSTs) from pigmented Vitis vinifera L. cell suspension cultures as putative anthocyanin transport proteins. J Exp Bot. 2008;59:3621-34

35. Mishiba K, Nishihara M, Nakatsuka T, Abe Y, Hirano H, Yokoi T, Kikuchi A Yamamura S. Consistent transcriptional silencing of 35S-driven transgenes in gentian. Plant J. 2005;44:541-56.

36. Hosokawa K, Matsuki R, Oikawa Y, Yamamura S. Production of transgenic gentian plants by particle bombardment of suspension-culture cells. Plant Cell Rep. 2000;19:454-8.

37. Aronesty E. Comparison of sequencing utility programs. Open Bioinform J. 2013;7:1-8.

38. Nakatsuka T, Nishihara M, Mishiba K, Yamamura S. Two different mutations are involved in the formation of white-flowered gentian plants. Plant Sci. 2005;169:949-58.

\section{Publisher's Note}

Springer Nature remains neutral with regard to jurisdictional claims in published maps and institutional affiliations. 